\title{
Knowledge of non-milk extrinsic sugars and general nutrition in children aged 10-12 years
}

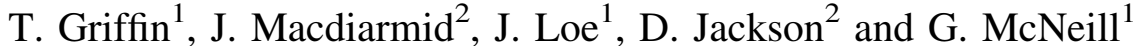 \\ ${ }^{1}$ Public Health Nutrition Research Group, University of Aberdeen, Aberdeen AB25 2ZP, UK and ${ }^{2}$ Rowett Institute \\ of Nutrition and Health, University of Aberdeen, Aberdeen AB21 9SB, UK
}

The average intake of non-milk extrinsic sugars (NMES) of children in Scotland is $17.4 \%$ of food energy ${ }^{(1)}$, which exceeds the Scottish dietary targets of $<10 \%$ of total energy for children ${ }^{(2)}$. A high intake of NMES poses a risk to dental health and has been linked to weight gain in children who consume a high volume of sugary beverages ${ }^{(3)}$. The aim of the present research was to ascertain children's knowledge of NMES and general nutrition knowledge as part of a bigger nutrition intervention, the Super Nutrition Advice for Kids (SNAK) project, which will measure whether an educational intervention improves children's knowledge of NMES and general nutrition.

Children ( $n$ 119, forty-five boys) aged 10-12 years from six primary schools completed a seven-item questionnaire. Five questions focused on sugar and nutrition (maximum score thirty-four marks), which had a Cronbach alpha of 0.73 (based on pilot work). Questions included recommended maximum daily intake of NMES, NMES content of a range of foods and general nutrition. Information on schools was collected, including percentage of pupils eligible for free school meals (FSM) and health-promoting school (HPS) status.

Children's results on the sugar and nutrition questions ranged from $10.3 \%$ to $61.8 \%$ (median 38.2 (interquartile range (IQR) 32.4-47.1) $\%)$. There was no significant difference in total knowledge score between boys and girls $(P=0.72)$, or between schools with different FSM eligibility $(P=0.76)$. Children from schools of committed HPS status scored significantly higher on the knowledge questionnaire compared to pupils from schools of commended status $(P=0.02)$. This finding may be a result of the specificity of the knowledge questionnaire compared with HPS campaigns that focus on a wider more general nutrition education. Of the children $89.1 \%$ knew the recommended intake of fruit and vegetables is 'five-a-day' whereas only $25.2 \%$ of children correctly identified what NMES are on a fouroption multiple-choice item. Children were asked what the maximum number of heaped teaspoons (tsp) of sugar (one tsp is $6 \mathrm{~g}$ sugar) a boy and girl aged 11-14 years could have in $1 \mathrm{~d}$ and how many teaspoons of sugar were in a range of common food and drinks. The results are shown in the Table highlighting that the majority of children tend to underestimate the amount of sugar in drinks compared with overestimations on food items.

\begin{tabular}{|c|c|c|c|c|c|c|c|}
\hline & $\%$ correct & $\%$ over-estimated & $\%$ under-estimated & Median (tsp) & IQR (tsp) & $\operatorname{Min}(\mathrm{tsp})$ & Max (tsp) \\
\hline \multicolumn{8}{|l|}{ Max recommended intake: } \\
\hline For a boy (ten tsp) & 6.7 & 10.9 & 82.4 & 5.0 & $3.0-8.0$ & 0 & 200 \\
\hline For a girl (eight tsp) & 1.7 & 14.3 & 84.0 & 4.0 & $2.0-6.0$ & 0 & 180 \\
\hline \multicolumn{8}{|l|}{ No. of tsp sugar in food and drinks: } \\
\hline Two digestive biscuits (one tsp) & 22.7 & 65.5 & 10.9 & 2.0 & $1.0-4.0$ & 0 & 25 \\
\hline $30 \mathrm{~g}$ Shreddies (one tsp) & 24.4 & 62.2 & 12.6 & 2.0 & $1.0-3.0$ & 0 & 35 \\
\hline $200 \mathrm{ml}$ orange juice $(3.5 \mathrm{tsp})$ & 19.3 & 10.9 & 69.7 & 2.0 & $1.0-3.0$ & 0 & 60 \\
\hline $300 \mathrm{ml}$ Fruit Shoot (five tsp) & 5.0 & 20.2 & 73.9 & 3.0 & $2.0-5.0$ & 0 & 40 \\
\hline $330 \mathrm{ml}$ can Irn Bru (six tsp) & 16.0 & 33.6 & 50.0 & 5.8 & $4.0-8.0$ & 1 & 80 \\
\hline
\end{tabular}

Min, minimum; Max, maximum.

The results show that children do not have a good understanding of NMES. The majority of children knew the recommendations for fruit and vegetable intake, showing that public health messages are being received, but children's knowledge on NMES was limited, highlighting an area for future health messages and research in this area.

1. Sheehy C, McNeill G, Masson L et al. (2008) Survey of sugar intake among children in Scotland. http://www.food.gov.uk/multimedia/pdfs/sugar intakescot2008rep.pdf

2. Scottish Government, Health and Community Care (2005) Scottish dietary targets for 2005. http://www.scotland.gov.uk/Topics/Health/health/19133/ 17756

3. Ludwig DS, Peterson KE \& Gortmaker SL (2001) Lancet 357, 505-508. 